\title{
METHODS FOR ORBIT OPTIMIZATION FOR THE LISA GRAVITATIONAL WAVE OBSERVATORY
}

\author{
GUANGYU LI* ${ }^{*} \|$, ZHAOHUA YI ${ }^{*}, \dagger$, GERHARD HEINZEL ${ }^{\ddagger}$ \\ ALBRECHT RÜDIGER ${ }^{\ddagger}$, OLIVER JENNRICH $^{\S}$, LI WANG $₫$, YAN XIA* \\ FEI ZENG* and HAIBIN ZHAO* \\ * Purple Mountain Observatory, Chinese Academy of Sciences, Nanjing 210008, P. R. China \\ $\dagger$ Department of Astronomy, Nanjing University, Nanjing 210008, P. R. China \\ ¥Max Planck Institute for Gravitational Physics, D-30167 Hannover, Germany \\ $\S$ European Space Research and Technology Center, 2200 AG Noordwijk (The Netherlands) \\ ฯ China Academy of Space Science, Beijing 100094, P. R. China \\ "Igyl@pmo.ac.cn
}

Received 31 December 2006

Revised 26 May 2007

Communicated by W.-T. Ni

\begin{abstract}
The Laser Interferometer Space Antenna (LISA) mission is a joint ESA-NASA mission for detecting low-frequency gravitational waves in the frequency range from $0.1 \mathrm{mHz}$ to $1 \mathrm{~Hz}$, by using accurate distance measurements with laser interferometry between three spacecraft, which will be launched around 2015 and one year later reach their orbits around the Sun. In order to operate successfully, it is crucial for the constellation of the three spacecraft to have extremely high stability. In this paper, several problems of the orbit optimization of the LISA constellation are discussed by using numerical and analytical methods for satisfying the requirements of accuracy. On the basis of the coorbital restricted problem, analytical expressions of the heliocentric distance and the trailing angle to the Earth of the constellation's barycenter are deduced, with the result that the approximate analytical solution of first order will meet the accuracy requirement of the spacecraft orbit design. It is proved that there is a value of the inclination of the constellation plane that will make the variation of the arm-length a minimum. The principle for selecting the optimum starting elements of orbits at any epoch is proposed. The method and programming principles of finding the optimized orbits are also presented together with examples of the optimization design.
\end{abstract}

Keywords: Coorbital restricted problem; LISA constellation; optimized orbit.

\section{Introduction}

The Laser Interferometer Space Antenna (LISA) mission is a joint ESA-NASA mission for detecting low-frequency gravitational waves in the frequency range from $0.1 \mathrm{mHz}$ to $1 \mathrm{~Hz}$. Three spacecraft will be launched around 2015, and reach their orbits around the Sun after one year. Observation will last for 5 to 10 years. In 2009, LISA Pathfinder will be launched to test the technology's feasibility. 


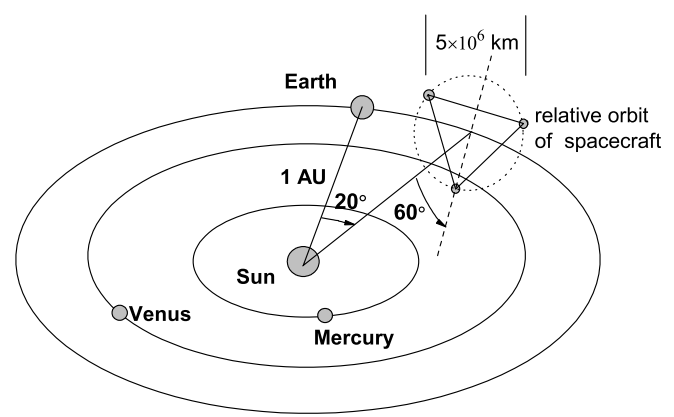

Fig. 1. LISA constellation moves around the Sun.

Distance variations between the spacecraft are measured with laser interferometry to detect gravitational waves. In order for LISA to operate successfully, it is crucial that the constellation of the three spacecraft have extremely high stability. As shown in Fig. 1, the three spacecraft form an equilateral triangle with an armlength (side length) of around $5 \times 10^{6} \mathrm{~km}$. The center of the constellation moves on the Earth orbit, trailing 20 degrees behind the Earth. The constellation plane is tilted $60^{\circ}$ out of the ecliptic. ${ }^{1}$

Due to the effect of the eccentricity of the spacecraft orbit and the gravity of the other bodies in the solar system including the Earth, and non-gravitational effects, ${ }^{2,3}$ the side length, the internal angles, the relative velocities among the spacecraft and the trailing angle between their barycenter and the Earth vary continuously. In order to ensure the precision of the distance measurements between the spacecraft, the variation of those parameters mentioned above should be at least within the given ranges shown in Table 1 according to the LISA requirements. Smaller variations may make simplifications in the hardware possible and consequently are highly preferred.

Thus, the problem of optimizing the orbits of the LISA constellation can be summarized as follows: finding a set of orbital elements $a_{i}, e_{i}, \omega_{i}, \Omega_{i}, I_{i}, M_{0 i},(i=$ $1,2,3)$ to make the following cost function take its minimum:

$$
Q\left(a_{i}, e_{i}, \omega_{i}, \Omega_{i}, I_{i}, M_{0 i}\right)=w_{1} \triangle D^{2}+w_{2} \triangle \alpha^{2}+w_{3} \triangle v_{r}^{2}+w_{4} \triangle \theta^{2}
$$

Table 1. The variation ranges of the parameters of the LISA constellation.

\begin{tabular}{lcc}
\hline Parameter & Average value & Permitted variation \\
\hline Arm-length $l$ & $5 \times 10^{6} \mathrm{~km}$ & $\triangle l= \pm 5 \times 10^{4} \mathrm{~km}$ \\
Internal angle $\alpha$ & $60^{\circ}$ & $\triangle \alpha= \pm 1.5^{\circ}$ \\
Relative velocity $v_{r}$ & 0 & $\triangle v_{r}= \pm 15 \mathrm{~m} / \mathrm{s}$ \\
Trailing angle to Earth $\theta$ & $20^{\circ}$ & $\triangle \theta$ small enough \\
\hline
\end{tabular}


with appropriately chosen weights $w_{j}$. The orbital elements are semi-major axis, eccentricity, argument of perihelion, ascending node, inclination and mean anomaly at the initial epoch, respectively. ${ }^{4}$

Since Faller ${ }^{5}$ et al. presented the concept of detecting gravitational waves by using laser ranging in space in 1985, research on the LISA constellation has been going on for many years. Vincent ${ }^{6}$ et al. (1987), Folkner ${ }^{7}$ et al. (1997), Cut$\operatorname{ler}^{8}$ (1998), Hughes ${ }^{9}$ (2002), Hechler ${ }^{10}$ et al. (2003), Dhurandhar ${ }^{11}$ et al. (2005), Sweetser ${ }^{12}$ (2005) and K. Rajesh Nayak ${ }^{13}$ et al. (2006) have investigated the LISA constellation from the viewpoint of science and spaceflight project. Because of the complexity of the space environment in which the LISA constellation moves, and the extremely high stability of the constellation required in the space project, further advanced research is expected.

In 1993, $\mathrm{Ni}^{14}$ et al. proposed the ASTROD mission concept. Some of its science objectives are similar to those of the LISA project, and they have been discussed at three international symposia in 2001, 2005 and 2006. Both LISA and ASTROD use interferometric laser ranging, and the Doppler effects on transmitted and received frequencies need to be addressed. LISA's strategy is to minimize arm-length variation and relative velocity of the spacecraft. For ASTROD, the arm-length changes of the three spacecraft are of the same order as the distances between the three spacecraft and the relative velocities go up to $70 \mathrm{~km} / \mathrm{s}$ with line-of-sight velocities varying from -20 to $+20 \mathrm{~km} / \mathrm{s}$. For $1064 \mathrm{~nm}(532 \mathrm{~nm})$ laser light, the Doppler frequency change goes up to 40 (80) GHz. For ASTROD, a strategy that relies on a different technology is used. The recent development of optical clocks and frequency synthesizers using optical combs makes this heterodyne problem tractable. ${ }^{15}$

Orbit design and simulation are two important parts of the pre-phase A study of ASTROD. Wei-Tou Ni, Chien-Jen Tang, Guangyu Li, and Yan Xia took part in the research successively ${ }^{16,17}$ which accumulated a wealth of experience for us to study the LISA orbits. In February 2006, Guangyu Li and Yan Xia were invited to visit the Max Planck Institute for Gravitational Physics and took part in research on the optimization of the LISA orbit design in cooperation with Gerhard Heinzel, Oliver Jennrich and Albrecht Rüdiger. This paper presents our results within this work so far.

In Sec. 2 of this paper, the selection of starting orbits for LISA for optimization is discussed. In Sec. 3, the motion of the barycenter of the constellation on the base of the plane coorbital motion is analyzed. In Sec. 4, the optimization algorithm and program structure that are used in this work are introduced briefly. In Sec. 5, the method to select the optimization orbits at any epoch is presented with examples.

\section{Selection of the Starting Orbits for Optimization}

The starting orbits is such a set of orbits that determines the starting point of the optimization trajectory in the orbit space, along which we can get to the targeted 


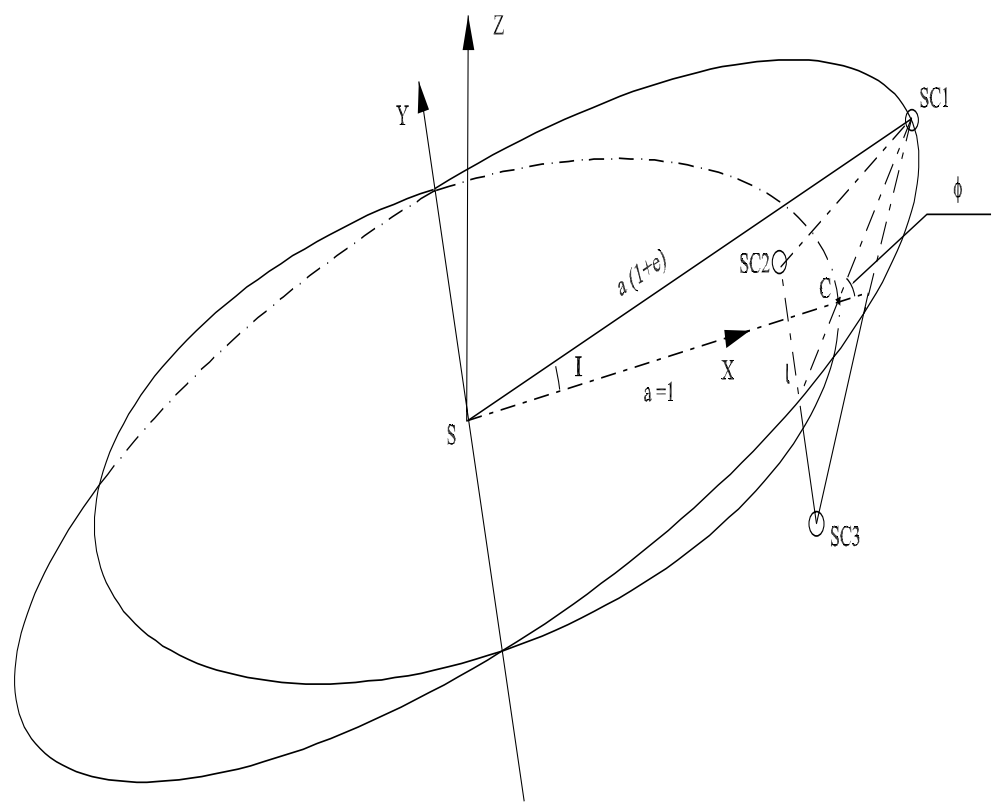

Fig. 2. Formation of the LISA constellation.

point and obtain the optimization orbits by given optimization algorithms, which have been discussed in detail in many papers. ${ }^{7,11-13} \mathrm{~A}$ brief review is given as the beginning of our discussion.

As is shown in Fig. 2, the Sun is located at point $\mathrm{S}$ while the horizontal circle is the orbit of the Earth, and three spacecraft SC1, SC2 and SC3 compose the LISA constellation, the barycenter of which, point $C$, moves along the orbit of the Earth coorbitally. ${ }^{18}$ In this paper, we assume that the masses of the three spacecraft are identical, so the barycenter of the constellation coincides with its geometrical center. The inclined ellipse is the osculating orbit of spacecraft SC1 at the initial epoch $t=0$, with $a, e, I$ and $f$ as semi-major axis, eccentricity, inclination to the ecliptic plane and true anomaly, ${ }^{4,19}$ respectively. Assuming spacecraft SC1 is located at its aphelion at the initial epoch, we have $f_{0}=M_{0}=\pi$ here, where $f_{0}$ and $M_{0}$ are the true anomaly and mean anomaly respectively at the time $t=0$. Defining $\phi$ as the angle between the constellation plane in which the three spacecraft move and the ecliptic plane, the important relationship between the eccentricity $e$, the inclination $I$ to the ecliptic plane of the spacecraft orbit, the average arm-length $l$ and the angle $\phi$ is expressed by the following equations:

$$
e=\sqrt{1+\frac{l^{2}}{3}+\frac{2 l}{\sqrt{3}} \cos \phi}-1,
$$

$$
\sin I=\frac{l \sin \phi}{\sqrt{3}(1+e)}, \quad \cos I=\frac{\sqrt{3}+l \cos \phi}{\sqrt{3}(1+e)} .
$$


Putting the Sun, S, at the origin, the ecliptic plane as the base plane and the direction from the origin to the projection of SC1 on the ecliptic plane as $x$-axis, a coordinate system, which is a non-inertial rotating coordinate system, is set up. Defining $\Omega$ and $\omega$ as the ascending node and the argument of perihelion ${ }^{4,19}$ of the spacecraft orbit in this system, the radius vector of SC1 can be expressed as

$$
\mathbf{r}=\mathbf{R}_{3}(\Omega) \mathbf{R}_{1}(I) \mathbf{R}_{3}(\omega+f)(r, 0,0)^{\tau},
$$

the superscript $\tau$ denoting the transpose operation of a matrix, $\mathbf{R}_{k}$ is the rotating matrix around the $k$ th axis $(k=1,3)$ :

$$
\mathbf{R}_{1}(\alpha)=\left(\begin{array}{ccc}
1 & 0 & 0 \\
0 & \cos \alpha & -\sin \alpha \\
0 & \sin \alpha & \cos \alpha
\end{array}\right), \mathbf{R}_{3}(\alpha)=\left(\begin{array}{ccc}
\cos \alpha & -\sin \alpha & 0 \\
\sin \alpha & \cos \alpha & 0 \\
0 & 0 & 1
\end{array}\right)
$$

As $I$ is a small quantity, 1 and $I$ could be regarded as the approximate values of $\cos I$ and $\sin I$, respectively. Therefore

$$
\mathbf{R}_{1}(I)=\mathbf{I}+I\left(\begin{array}{ccc}
0 & 0 & 0 \\
0 & 0 & -1 \\
0 & 1 & 0
\end{array}\right)
$$

where $\mathbf{I}$ is the unit matrix. Substituting (7) into (5) and simplifying it, we get

$$
\mathbf{r}=r(\cos (\Omega+\omega+f), \sin (\Omega+\omega+f), \sin (\omega+f) I)^{\tau} .
$$

From Fig. 2, we can see that both the $\Omega$ and $\omega$ of the spacecraft SC1 equal to $3 \pi / 2$. Then at $t=0$,

$$
\Omega+\omega+M_{0}=0(\bmod 2 \pi) .
$$

To form the constellation, it is a necessary condition for all the three spacecraft that each of their orbits keeps the relationship described in Eq. (8). Numerical calculation indicates that the better way to select the other two orbits is:

(i) Rotating the orbit of SC1 around the $z$-axis by $\pi / 3$ and $2 \pi / 3$, respectively, to form the orbits of SC2 and SC3;

(ii) Adjusting the mean anomalies of SC2 and SC3 to keep the relationship (8).

Thus the five intial orbit elements of the three spacecraft for optimization are shown in Table 2. The unit of the semi-major axis $a$ is the astronomical unit AU.

From Eq. (8), we gain

$$
\Omega+\omega+f=f-M_{0}
$$

Substituting it into expression (7), we get

$$
\mathbf{r}=r\left(\cos \left(f-M_{0}\right), \sin \left(f-M_{0}\right),-\cos (f) I\right)^{\tau} .
$$


Table 2. The 5 starting orbit elements of the three spacecraft for optimization.

\begin{tabular}{cccccc}
\hline $\mathrm{i}$ & $\mathrm{a}$ & $\omega$ & $\Omega$ & $M_{0}$ & $f_{0}$ \\
\hline $\mathrm{SC} 1$ & 1 & $270^{\circ}$ & $270^{\circ}$ & $180^{\circ}$ & $180^{\circ}$ \\
$\mathrm{SC} 2$ & 1 & $270^{\circ}$ & $30^{\circ}$ & $60^{\circ}$ & $60^{\circ} .963335$ \\
$\mathrm{SC} 3$ & 1 & $270^{\circ}$ & $150^{\circ}$ & $300^{\circ}$ & $299^{\circ} .036665$ \\
\hline
\end{tabular}

It is necessary to point out that the starting formation of the constellation achieved above is not a strictly equilateral triangle and will not become such a perfect triangle ever in its future evolution. However, if begun from this starting formation, the optimization formation will be achieved much more easily.

The next step is to discuss the variation of the arm-length (side length) of the constellation in the frame of two-body motion. Although that is far from the real motion with perturbation, it can still provide an important inspiration for our work. For analysis convenience the three spacecraft are differentiated by subscripts $1,2,3$. So from Eq. (10), the vector from SC1 to SC2 is

$$
\mathbf{r}_{12} \equiv \mathbf{r}_{2}-\mathbf{r}_{1}=\left(\begin{array}{c}
r_{2} \cos \left(f_{2}-M_{20}\right)-r_{1} \cos \left(f_{1}-M_{10}\right) \\
r_{2} \sin \left(f_{2}-M_{20}\right)-r_{1} \sin \left(f_{1}-M_{10}\right) \\
\left(r_{1} \cos f_{1}-r_{2} \cos f_{2}\right) I
\end{array}\right)
$$

Hence the square of the arm-length is

$$
\begin{aligned}
r_{12}^{2}= & \left(\mathbf{r}_{2}-\mathbf{r}_{1}\right) \cdot\left(\mathbf{r}_{2}-\mathbf{r}_{1}\right)=r_{1}^{2}+r_{2}^{2} \\
& -2 r_{1} r_{2} \cos \left[\left(f_{1}-M_{10}\right)-\left(f_{2}-M_{20}\right)\right]+\left(r_{1} \cos f_{1}-r_{2} \cos f_{2}\right)^{2} I^{2} .
\end{aligned}
$$

From the equation of the center ${ }^{4,19}$ we have

$$
f_{i}-M_{i}=\left(2 e-\frac{1}{4} e^{3}\right) \sin M_{i}+\frac{5}{4} e^{2} \sin 2 M_{i}+\cdots .
$$

We define the angle $f_{12}$ as the difference

$f_{2}-M_{20}-\left(f_{1}-M_{10}\right)=\left(2 e-\frac{1}{4} e^{3}\right)\left(\sin M_{2}-\sin M_{1}\right)+\frac{5}{4} e^{2}\left(\sin 2 M_{2}-\sin 2 M_{1}\right)+\cdots$,

which is a small quantity of the same order as $e$. With the accuracy of terms up to $e^{2}, \cos f_{12}$ could be approximated as:

$$
\cos f_{12}=1-\frac{f_{12}^{2}}{2}
$$

Noting that ${ }^{4,19} r_{i}=1-e \cos E_{i}, r_{1}-r_{2}=e\left(\cos E_{2}-\cos E_{1}\right) \approx e\left(\cos M_{2}-\cos M_{1}\right)$, where $E_{i}$ is the eccentric anomaly of the $i$ th spacecraft, Eq. (12) becomes

$$
r_{12}^{2}=12 e^{2}+3\left(\sin ^{2} I-3 e^{2}\right) \sin ^{2}\left(M_{1}-\frac{2 \pi}{3}\right) .
$$

Similarly we can get the two Equations for $r_{23}^{2}$ and $r_{31}^{2}$.

The formation of the constellation is not always a strictly perfect equilateral triangle, so that $\sin ^{2} I-3 e^{2} \neq 0$. Let $l^{2}=12 e^{2}$, namely $l=2 \sqrt{3} e$, where $l$ is 


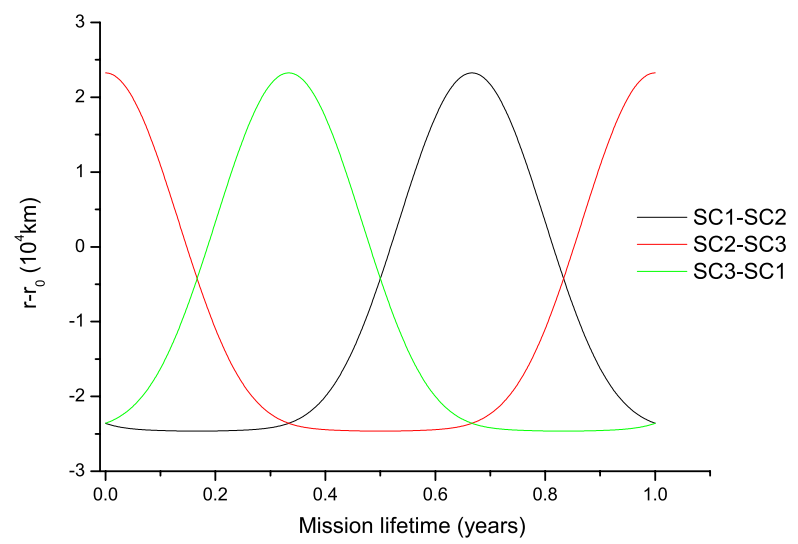

Fig. 3. Variation of the arm-length when $\phi=60^{\circ} .4776$.

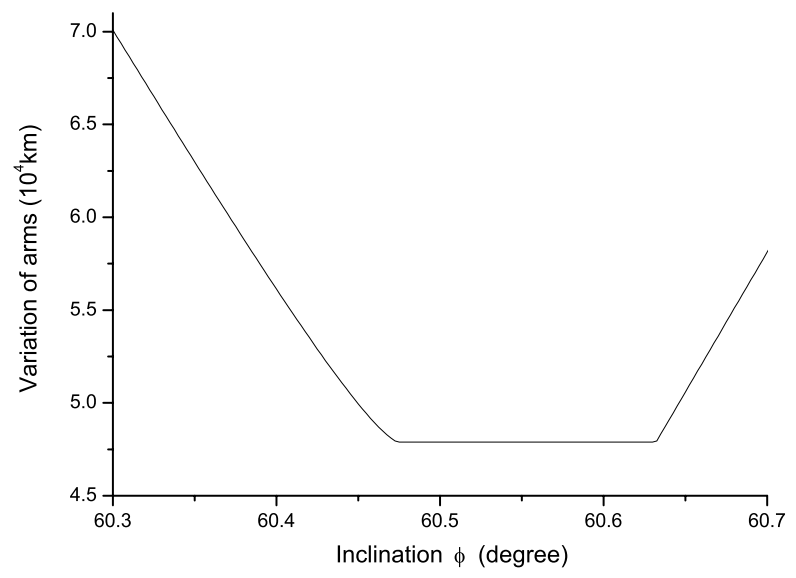

Fig. 4. The relationship between the inclination $\phi$ and the variation of the arm-length $\Delta$.

the average arm-length. From Eq. (2) we have $\cos \phi=\frac{1}{2}-\frac{\sqrt{3}}{8} l=0.492764(l=$ $0.03342292561 \mathrm{AU})$ which gives $\phi=60^{\circ} .4776$ and the variation of the arm-length is shown as Fig. 3. Numerical calculation indicates that it is also the best value of the inclination of the constellation plane, which makes the arm-length variation approach a minimum in the frame of the two-body problem.

Numerical analysis also indicates that when $60^{\circ} .47<\phi<60^{\circ} .63$, the variation of the arm-length keeps at the minimum around $4.788 \times 10^{4} \mathrm{~km}$, as shown in Fig. 4. Using a different method, K. Rajech Nayak et al. drew the nearly identical conclusion. ${ }^{13}$ 
Assuming $\phi=60^{\circ} .4776$, from Eqs. (2) and (3), the values of the eccentricity $e$ and the inclination $I$ of the spacecraft orbits are

$$
\begin{aligned}
& e=0.0096483717, \\
& I=0^{\circ} .95292153=0.016631618 \mathrm{rad} .
\end{aligned}
$$

The other elements have been given in Table 2, determining the starting orbits for the optimization.

\section{Motion of the Barycenter of the LISA Constellation}

The approximate analytical expressions of the argument $\theta$ and the heliocentric distance $r$ of the barycenter of the LISA constellation have been presented in another paper, ${ }^{18}$ which will be sketched briefly for orbit optimization in this section.

Due to the requirement of orbit design, the barycenter $C$ is always trailing behind the Earth, namely the initial argument $\theta_{0}<0$. Hence $\sin \frac{\left|\theta_{0}\right|}{2}=-\sin \frac{\theta_{0}}{2}$. Putting the expressions into different forms gives

$$
\begin{gathered}
r=\frac{1+2 k n t-\frac{3}{32} k k_{1}(n t)^{3}}{1+e \sin (\Delta \theta+n t)}, \\
\Delta \theta=-\frac{3}{2} k(n t)^{2}+2 k^{2}(n t)^{3}+\frac{9}{256} k k_{1}(n t)^{4},
\end{gathered}
$$

where $e=5 \times 10^{-5}, n$ is the mean motion ${ }^{4,19}$ (average angular velocity) of the barycenter, $t$ is the mission lifetime in days, and

$$
\begin{gathered}
k=\mu \sin \theta_{0}\left(1+\frac{1}{8} \csc ^{3} \frac{\theta_{0}}{2}\right), \\
k_{1}=\mu\left[8 \cos \theta_{0}-\csc \frac{\theta_{0}}{2}\left(1+2 \cot ^{2} \frac{\theta_{0}}{2}\right)\right],
\end{gathered}
$$

where $\mu=m_{e} /\left(m_{e}+m_{s}\right)=3.04 \times 10^{-6}, m_{s}$ and $m_{e}$ is the Sun mass and the Earth mass, respectively.

On the base of the above equations the following conclusions about the motion of the barycenter of the LISA constellation can be drawn:

(i) In the classical result of celestial mechanics, ${ }^{4,19}$ the Lagrange point $L_{5}$ is at $\theta_{0}=-60^{\circ}$, and $k, \triangle \theta$, and $\triangle r$ all vanish if $\mathrm{C}$ is at that location.

(ii) The parameter $k$ given in Eq. (19) describes the orbit instability. The relationship between $k$ and $\theta_{0}$ is shown in Fig. 5. When $\theta_{0}$ increases from $-60^{\circ}$, $k$ starts its monotonic increase slowly (note the logarithmic scale). As soon as $\theta_{0}>-5^{\circ}$, when the spacecraft are quite close to the Earth, $k$ begins to increase rapidly, and from Eq. (18) the absolute value of $\Delta \theta$ increases even more rapidly. Consequently the orbits are much too unstable for such a small 


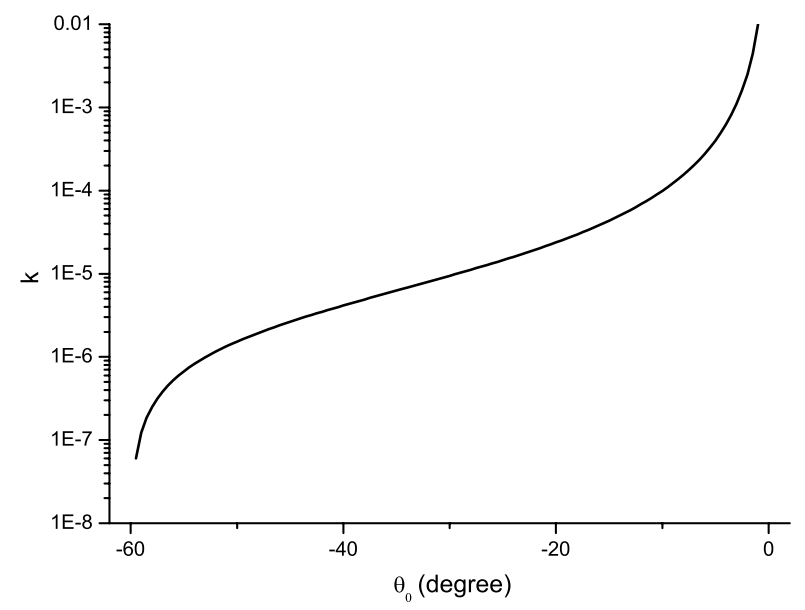

Fig. 5. The relationship between the parameter $k$ and the initial argument $\theta_{0}$.
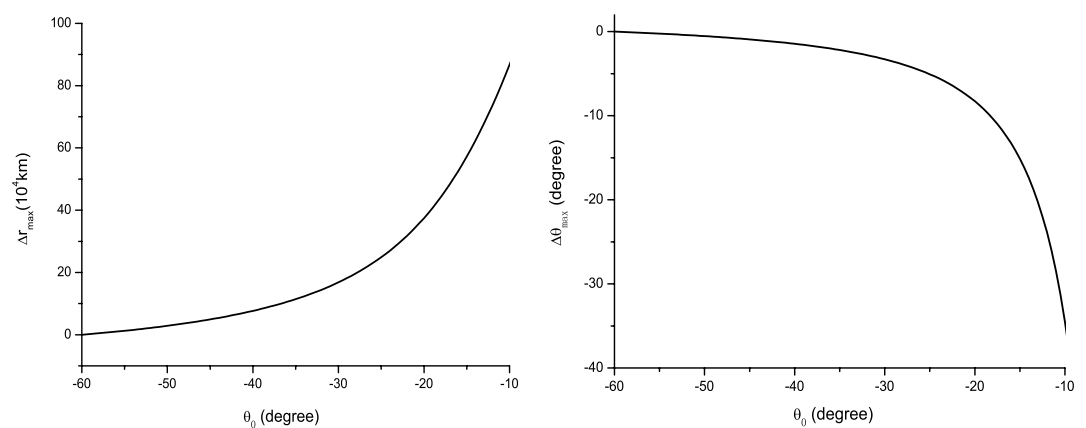

Fig. 6. The dependence of the maximum variations $\Delta r$ and $\Delta \theta$ on the initial argument $\theta_{0}$.

value of $\theta_{0}$, so henceforth we consider only cases within the parameter range $\theta_{0} \in\left[-60^{\circ},-10^{\circ}\right]$.

(iii) For $r_{0}=1 \mathrm{AU}$, in our interesting parameter range $\theta_{0} \in\left[-60^{\circ},-10^{\circ}\right]$, the argument $\theta$ and the increment $\Delta r$ of the heliocentric distance are nearly monotonic functions of time $t$. Thus their absolute value will reach the maximum at the end of the mission lifetime $(t=3700$ days $)$. The two diagrams in Fig. 6 show the relation between the maximum values and the initial argument $\theta_{0}$.

(iv) If $\theta_{0}=-20^{\circ}, \Delta \theta_{\max }=-7^{\circ} .85$ and $\Delta r_{\max }=36 \times 10^{4} \mathrm{~km}$ are the solutions. With increasing time, the heliocentric distance becomes larger and larger. Selecting $\theta_{0}$ with a larger absolute value, for example, $\theta_{0}=-30^{\circ}$, would significantly decrease $\Delta r$ and $\Delta \theta$. But due to the technical and other reasons, the value near $-20^{\circ}$ would be a preferable choice in the LISA mission. ${ }^{1}$ Although 
the value $\Delta r$ and $\Delta \theta$ here are larger by about an order of magnitude than the target value given in Table 1 , taking these elements as a starting point for orbit optimization, we will still reach our goal in the end. That will be discussed in detail in Secs. 4 and 5.

(v) Along with the increase of the heliocentric distance, the mean motion $n$ decreases consequently, which results in the increase of the earth-trailing angle. If $r_{0}$ is slightly smaller than 1.0 AU by selection, it will gradually increase to 1.0 AU in the mission lifetime, which results in an effective decrease of the variation range of the angle $\theta$. The equation $\Delta r=-2 \Delta n /(3 n)$ can be used to estimate an initial parameter $r_{0}$ for the numerical optimization. Figure 7 shows an optimized result where the variation range of the argument $\theta$ is below $2^{\circ} .6$ when $\theta_{0}=-22^{\circ}$ and $r_{0}=0.9996$ AU. During 80 percent of the mission lifetime, the variation $\Delta \theta$ is less than $0.5^{\circ}$. But the variation range of $\Delta r$ is larger than before, about $80 \times 10^{4} \mathrm{~km}$. In the practical optimization process a balance between the two variations is needed. It will be discussed in Sec. 5.4.
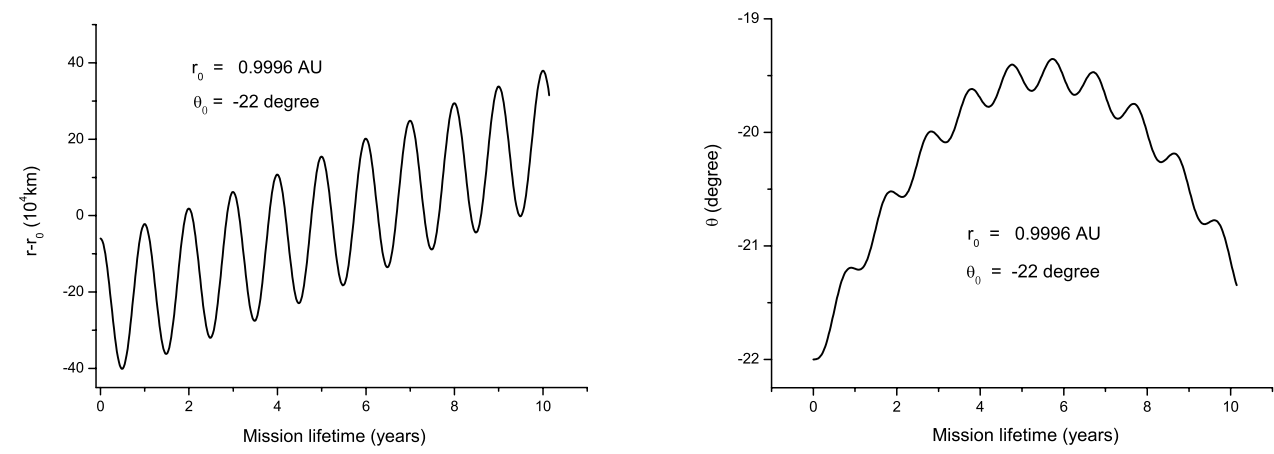

Fig. 7. An optimization result for the initial trailing argument $\theta_{0}$.

(vi) The solar-radiation pressure is a significant perturbation force for the spacecraft. ${ }^{3,20,21}$ Specifically, the area-to-mass ratios of the spacecraft are typically in the range from $10^{-3}$ to $10^{-2} \mathrm{~m}^{2} / \mathrm{kg}$. Thus, to a rough first approximation, a spacecraft in orbit about the Sun behaves as if the Sun's gravitational constant GM were reduced by the fractional amount $10^{-6}$ to $10^{-5}$. This requires, inter alia, that the semi-major axis of the LISA spacecraft be smaller than that of the Earth by about $1 / 3$ of this amount, $3 \times 10^{-7}$ to $3 \times 10^{-6} \mathrm{AU}$, if the mean motion of the spacecraft is to match that of the Earth. However, the actual sizes and also the actual directions of the forces due to radiation pressure depend on the specifics and are very complicated, since they depend on the reflectivities, shapes, and orientations of the various surfaces of the spacecraft. ${ }^{22,23}$ Moreover, back-reaction from blackbody radiation emitted from the spacecraft may possibly need to be taken into account. ${ }^{24}$ This effect depends 
on differential heating and cooling of the spacecraft surfaces, which depend in turn on the thermal properties of all parts of the spacecraft. Although all these non-gravitational effects on the spacecraft would not influence the orbits of LISA spacecraft due to their drag-free motion, modeling these effects a priori to the accuracy contemplated in our work would be a challenging task for the future.

\section{Optimization Algorithm and Program Structure}

Now it is time to discuss the problem of orbit optimization. Based on the strategy of separate calculation, the three orbits are optimized successively, so that only one orbit is optimized at a time, determined exclusively by the six starting orbit elements $^{4} a, e, \omega, \Omega, I, M_{0}$. The problem will be discussed in the following subspace of the orbital space of six dimensions

$$
\left\{x_{i 0}-g_{i} \leq x_{i} \leq x_{i 0}+g_{i}, i=1,2, \ldots, 6\right\}
$$

in which a point $\mathbf{x}=\left(x_{1}, x_{2}, \ldots, x_{6}\right)^{\tau}$ presents an orbit and the six coordinates $x_{i}$ are the six orbital elements of which the special denotation stated before will not be used any more. The cost function (1) becomes a function of the point $\mathbf{x}$. It is easy to prove that if $|\Delta l|<5 \times 10^{4} \mathrm{~km}$, the inequalities $|\Delta \alpha|<1^{\circ} .5$ and $\left|\Delta v_{r}\right|<15 \mathrm{~m} / \mathrm{s}$ are certainly valid. Therefore the cost function can be simplified to

$$
Q(\mathbf{x})=w_{1} \Delta l^{2}+w_{2} \Delta \theta^{2}
$$

The Hybrid Reactive Tabu Search(C-RTS) algorithm ${ }^{25}$ presented by R. Battiti and G. Techiolli is applied to our orbit optimization. This section gives an outline of the algorithm and the processes in it that could be applied to the orbit optimization problem.

\subsection{Local optimization algorithm}

The Affine Shaker algorithm shown in Fig. 8 is applied to resolve the optimal local minimum. During the initialization stage, the initial base matrix is generated as

$$
\mathbf{B}(0)=\frac{1}{4}\left(\begin{array}{cccc}
g_{1} & 0 & \ldots & 0 \\
0 & g_{2} & \ldots & 0 \\
\ldots & \cdots & \ldots & \ldots \\
0 & 0 & \ldots & g_{6}
\end{array}\right)
$$

its column vectors $\mathbf{b}_{j}(t)$ named base vectors.

The search starts from the starting point $\mathbf{x}(0)$ selected randomly and is iterated with $t$ as loop count variable until the cost function satisfies the preset condition, or the loop count passes the preset upper limit. In each loop a random displacement 
vector is generated first:

$$
\boldsymbol{\delta}=\sum_{j=1}^{6} \operatorname{rand}_{j} \mathbf{b}_{j}(t-1),
$$

where $0<\operatorname{rand}_{j} \leq 1$ are random numbers.

In this way, two new points $\mathbf{x}^{+}(t)=\mathbf{x}(t-1)+\boldsymbol{\delta}(\mathrm{t})$ and $\mathbf{x}^{-}(t)=\mathbf{x}(t-1)-\boldsymbol{\delta}(\mathrm{t})$ are generated for selection. First the point $\mathbf{x}^{+}(t)$ is tested. This first shot is successful only if this point is better than the point $\mathbf{x}(t-1)$. Then, if the first shot is not successful, the point $\mathbf{x}^{-}(t)$ is tested, which is named second shot. As long as a shot is successful in any of the two tests, the search point is moved to the test point and the affine transformation matrix is generated:

$$
\mathbf{P}=\mathbf{I}+(\rho-1) \frac{\boldsymbol{\delta} \boldsymbol{\delta}^{\tau}}{\boldsymbol{\delta}^{\tau} \boldsymbol{\delta}},
$$

where $\rho>1$, to expand the search range. If neither of the two shots was successful, the search point is not moved any further, but the affine transformation matrix is still generated in the case that $\rho<1$ to limit the search range. At the end of each iterative loop, the base matrix is updated as

$$
\mathbf{B}(t)=\mathbf{P B}(t-1)
$$

and the loop count variable $t$ is increased by 1 .

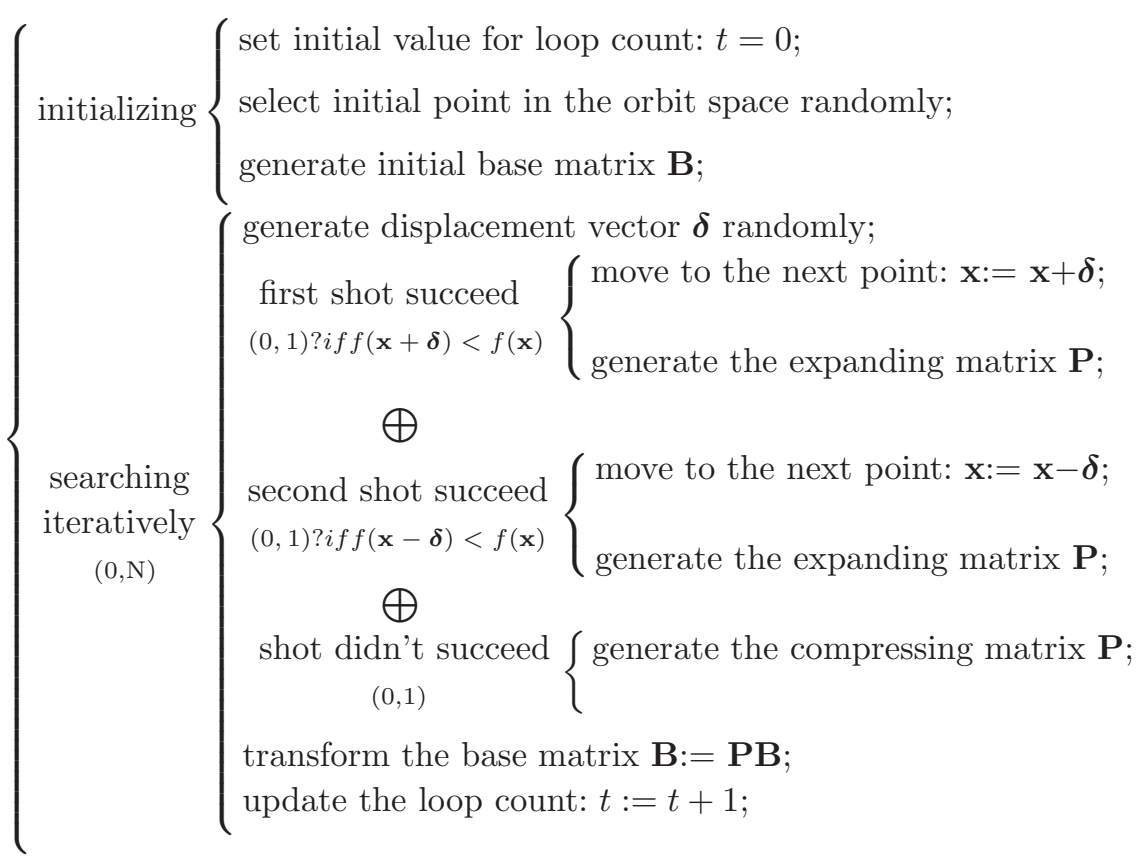

Fig. 8. Flow chart of Affine Shaker algorithm. 


\subsection{Global optimization algorithm}

Due to the complexity of the problem, there are probably many optimal local minima in the orbital space (22), which makes the above local optimization algorithm get stuck in a certain local point and never search for a global optimization point. This problem can be solved by the global optimization algorithm, C-RTS. ${ }^{25}$

The algorithm is a kind of iterative search method. The global optimal problem then could be broken down into the local optimal problems. The key point of the algorithm is to ensure that the search trajectory covers all of the global region. The intensified-search, the diversified-search and the prohibition period parameter are the three major elements of the algorithm which will be illustrated later. Now we sketch the algorithm in two parts: data structure and search algorithm.

\subsubsection{Data structure}

First, we should search for an appropriate data structure for the problem to discretize our continuous problem of the orbit optimization and establish an appropriate algorithm. Because the initial search space (22) is a six-dimension space, which can be organized in the structure of a multiway tree of boxes with 64 children for searching expediently.

For simplicity, a 2-dimensional example is used to describe the terminology which will be used in our algorithm. Assuming the searching is limited within the rectangular region (or box) $\left\{x_{i 0}-g_{i} \leq x_{i} \leq x_{i 0}+g_{i}, i=1,2\right\}$, the region is named root-box, which is a subset of the orbital space (22). Halving the root-box on each dimension, the space can be divided into $2^{2}=4$ equal-sized children, which are named child boxes of the root-box. The total of the child boxes cover the root-box. The root-box divided is named parent-box now. The position of a child box in its parent box can be identified by a 2-bit binary number (namely a decimal integer less than 4). The first bit stands for the position of the 1st side. If it equals to zero, the left half divided in the parent box is selected. Otherwise the right half is selected. Similarly, the second bit stands for the position of the second side. As shown in the left diagram of Fig. 9, the identifiers of the 4 child boxes that are the division of the root-box are $00,01,10,11$, respectively.

One of the aforementioned four boxes is selected stochastically as the starting point for the global optimum search. Once two different local minima are found in one child-box during the search, the child-box is then subdivided in the aforesaid way immediately. Assuming the child-box with the identifier of 10 is divided, 4 smaller child-boxes will be generated. Their identifiers relative to their parent-box 10 are still $00,01,10,11$, respectively, while their absolute identifiers, namely the position relative to the root-box are 1000, 1001, 1010 and 1011. The higher 2 bits are the identifer of the parent-box and the lower 2 bits are the identifers of the child-boxs, shown as the right diagram of Fig. 9. 

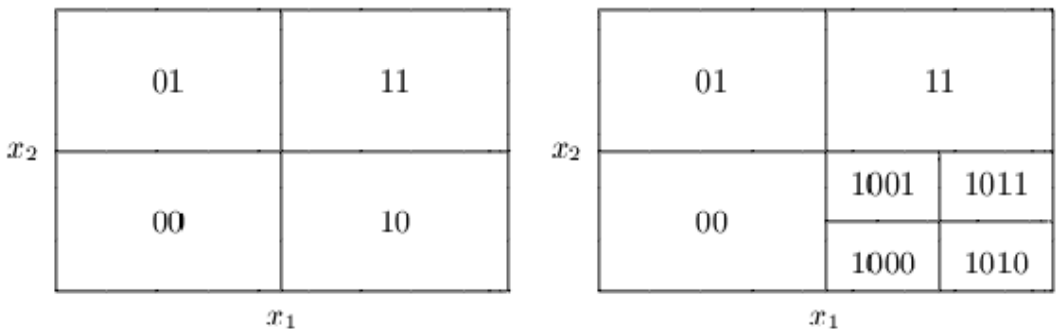

Fig. 9. Dividing a 2-dimension region, boxes and their identifiers.

Hereafter, the global optimum is searched within the set of 7 undivided boxes with identifier 00, 01, 11, 1000, 1001, 1010, 1011, respectively, which is named leaf-box. The depths of the leaf-boxes are different. The depth of the first three leaf-boxes are 0 , while the others are 1 . The further the searching and dividing is going, the larger is the depth of the leaf-box. Therefore, the data structure of the multiway tree of boxes with 4 children is built up now.

By inverting any bit of the identifier of a box, the box obtained is adjacent to the primary box. For example, the neighbors of the box 00 are 10 and 01 . At each step, the search moves to an appropriate neighboring box, which is named the node of the search trajectory. If the current node is box 10, which no longer exists because of the division, one of the 4 child-box of the box 10 is selected randomly as the node replacement. Vice versa, if the current node is box 1001 and the next node will be 0001 which is also not existing because of the non-division of box 00, the new node replacement will be box 00 . All the nodes generated during the successive search can be linked into a trajectory according to their generation order.

As soon as a leaf-box emerges during the search, a point in it is selected stochastically where the value of cost function is calculated by the method in Sec. 5.3 and is regarded as the evaluation of the box. When the box emerges again, another random point in it is selected and the value of the cost function at this point is also calculated. If this value is better than the earlier evaluation of the box, it will be regarded as the new evaluation. Otherwise, the evaluation will remain unchanged. The problem is discretized in this way.

Then back to the orbit optimization problem itself, the difference is merely that the orbital space $(21)$ is a 6 -dimension space. $2^{6}=64$ child-boxes will be produced by the division of a box. The position of a child-box in its parent-box can be identified with a 6-bit binary number (namely a decimal integer less than 64). The position of the $i$ th side is determined by the $i$ th bit of the binary number. If it equals to zero, the left half of the parent box is selected. Otherwise the right half is selected. The successive search will build up a data structure of the multiway tree of boxes with 64 children. 


\subsubsection{Searching algorithm}

The flow chart of the global optimization algorithm is shown in Fig. 10. The algorithm is composed of two parts: initializing and searching iteratively. The tasks of initializing are to set up the initial values of the relative parameters and to select the starting point of the search trajectory stochastically. The search is iterated until the cost function satisfies the preset condition, or the loop count overruns the preset upper limit. In each of the iterations, the parameters, such as prohibition period etc, are modified according to the condition of the time at first, and the searching mode is determined subsequently. The prohibition period is an important parameter for this algorithm, as it helps the searching trajectory to avoid running into endless loops. This algorithm has two search modes: the intensified-search which moves to the leaf-box in the neighborhood with optimum evaluation to find the global minimum, and the diversified-search which moves to a random neighbor leaf-box to avoid running into a local minimum. The algorithm follows the three rules shown below:

(i) If the evaluation of the current leaf-box is better than the evaluations of all the neighbor leaf-boxes in the intensified-search, the Affine Shaker algorithm in Sec. 4.1 is activated to find a local minimum.

(ii) Once two different local minima are found in one leaf-box, the box is divided by the method in the previous subsection.

(iii) Moving from a leaf-box to another visited one in the prohibition period is prohibited. And the prohibition period is decided based upon the condition of that time.

A detailed description of this algorithm is presented in Ref. 25.

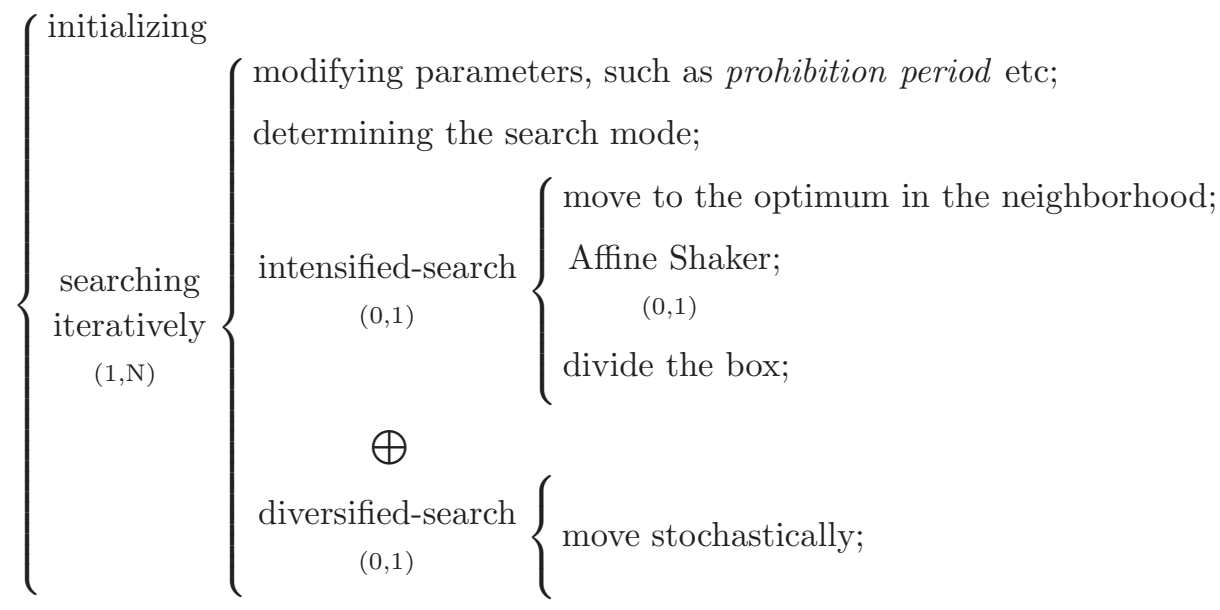

Fig. 10. Flow chart of the global optimization algorithm. 


\section{Examples of Optimized Orbits}

On the base of the above sections, for any given initial epoch the optimization process for the selected starting orbits of spacecraft can be repeated time after time by using the program of the optimization algorithm until the ideal orbits are achieved. In this section, first the determination of the starting orbits for optimization will be discussed; then the ephemeris framework and the integrator used in the optimization and a briefly precision analysis will be presented; at last, three examples will be given as the results of the methods of this paper.

\subsection{Determination of the starting orbits for optimization}

The starting orbits referred to the rotating coordinate system for the optimization of the three spacecraft have been given in Sec. 2. The four elements $a, \omega, \Omega, M_{0}$ are listed in Table 2, two elements $e, I$ are given by expression (16). It is necessary for calculating the orbits evolution by numerical integration, to translate the starting orbits to the heliocentric ecliptic coordinate system which can be realized through only translating the ascending node $\Omega$ by the following equation and keeping the other elements invariable.

$$
\Omega=\Omega_{r}+\Omega_{e}+\omega_{e}+M_{e}+\theta_{0},
$$

where $\Omega_{r}$ is the ascending node of the spacecraft referred to the rotating coordinate system, $\Omega_{e}, \omega_{e}, M_{e}$ are the ascending node, the perihelion argument, the mean anomaly of the Earth orbit referred to the J2000.0 heliocentric ecliptic coordinate system, respectively, and $\theta_{0}$ is the argument angle of the barycenter of the constellation to the Earth, which is positive when leading the Earth, negative when trailing.

\subsection{Numerical integration and its precision}

Because the mission lifetime is about 10 years, all the orbits of the spacecraft are integrated numerically from the time of the initial epoch through 3,700 days. The number of perturbing bodies for the integration can be adjusted according to the real situation. For example, either the perturbation of the Earth-Moon system only is considered for estimation at first and the perturbation of other planets is considered for accurate calculation in the end; or the perturbation of all the bodies is considered from the beginning to the end. Since the accuracy of the spacecraft position is required to be around thousands of kilometers in the mission lifetime, the perturbation from asteroids and general relativity can be ignored. All those perturbation effects should be considered during the future more accurate research. Although the non-gravitational effects on the orbits are important sources of perturbation, ${ }^{2,3}$ they also can be ignored due to the drag-free motion of the spacecraft.

The positions of planets, the Sun, the Moon and Pluto for the integration are gotten from PMOE 2003 ephemeris frame-work,${ }^{26}$ completed by Guangyu Li et al., 
of which the prediction accuracy of the planet position is the same as that of JPL DE 405 ephemeris.

The Cowell-Zhang integrator, ${ }^{27}$ which is completed by Jiaxiang Zhang by improving the classical Cowell's method, ${ }^{4,28}$ is selected for the numerical integration with 0.25 days step width. The accumulation errors of this integrator has three main sources:

The first one is the rounding error, generated by adding the small quantity (right-hand function) and the big quantity (old summation) during the extrapolation for generating the new summation. On the base of the theory of numerical integration, after $n$ steps of extrapolation the probable error is about ${ }^{4} 0.1124 n^{3 / 2}$, in units of the last decimal. Taking 0.25 days as the step width and integrating through 144,000 steps (about 98 years), the probable error is about 6,142,003, that is to say the error is passed 7 digits forward. If the step length is 0.125 day, it is 8 digits. The effective digits of the floating point numbers with 10 bytes are 19 decimal digits, which is not long enough to avoid a serious accumulation of this kind. If the data structure of double precision is introduced, the effective digits of the summation will increase to 26 decimal digits. In this case, the accumulation of the rounding error, generated by insufficient word length, would be negligible.

The second source is the truncation error of the approximate expressions in use. In our integrator, the truncation error of the coordinates integrated in a step is proportional to the product of the 8th order differences of the right-hand function and the 8th power of the step width. If an appropriate step width is selected, this kind of error can be decreased to become negligible.

The last source is the rounding error generated in the right-hand function calculation process, which can be decreased by increasing the effective bits of the initial values and the word length of the variables in the program. Using the variables of floating numbers with 10 bytes and initial value of 16 decimal digits, the total accumulation error is controlled to within a reasonable range.

\subsection{Calculation of the cost function}

Due to the three orbits of the spacecraft being optimized independently one by one in our algorithm, during each time of the optimization, only the one orbit that is being optimized and is given by a point $\mathbf{x}$ in the orbital space (22), is variable, while the other two orbits are kept constant. All three orbits of the spacecraft are integrated numerically from the time of the initial epoch through 3,700 days. During the integration the heliocentric ecliptic coordinates of the spacecraft $\mathbf{r}_{i}=$ $\left(x_{i}, y_{i}, z_{i}\right)^{\tau}(i=1,2,3)$ can be obtained, and the three arm-lengths $r_{i j}=\mid \mathbf{r}_{j}$ $\mathbf{r}_{i} \mid(i, j=1,2,3 ; j>i)$ and the trailing angle $\theta$ of the barycenter of the constellation to the Earth can be calculated. After the integration, the maximum variation of the three arm-lengths $\Delta l$ and the maximum variation of the trailing angle $\Delta \theta$ (See Fig. 11) can be derived. Then the cost function $Q(\mathbf{x})$ can be calculated by Eq. (22). 


\subsection{Optimized orbits}

As an example, the barycentric dynamical time (TDB) $t_{0}=2457023.5$, namely 1 January, 2015, is set as the initial epoch, then the ascending node, the perihelion argument and the mean anomaly of the Earth orbit, referred to the J2000.0 heliocentric ecliptic coordinate system, are, respectively:

$$
\begin{gathered}
\Omega_{e}=138.056381225628^{\circ}, \\
\omega_{e}=326.19176964486^{\circ}, \\
M_{e}=355.911260986297^{\circ} .
\end{gathered}
$$

If a different initial epoch were selected, only those three elements should be changed, the below treatment is still available. Usually the minor adjustment to the semi-major axis $a$ of the starting orbits and the argument $\theta$ of the barycenter of the constellation to the Earth will improve the optimized results. This will be illustrated concretely with the following three examples.

Example 1. Using the starting orbital elements determined in Sec. 2, assuming $\theta_{0}=-22^{\circ}$, and modifying the ascending node by relation (27) provides the following starting orbits (see Table 3). The unit of the semi-major axis is AU and the unit of the angle elements is degree. Due to the influence of the perturbation, the argument angle $\theta_{0}$ is set to $-22^{\circ}$ rather than $-20^{\circ}$, to keep it at a reasonable value during the mission lifetime.

The optimized orbit elements are shown in Table 4. The initial positions and velocities of the three spacecraft are shown in Table 5 . The variation range of the three arm-lengths and the trailing angle before and after optimization are shown in Tables 6 and 7 .

Example 2. In the previous example, after optimization the variation range of the trailing angle of the barycenter of the constellation, which is $9.3^{\circ}$, is a little larger. Based on the conclusion mentioned in Sec. 3, decreasing the semi-major axis of the starting orbits is used to reduce the angle. In this example, $a=0.9996$,

\begin{tabular}{|c|c|c|c|c|c|c|}
\hline & $\mathrm{a}$ & e & $\omega$ & $\Omega$ & I & M \\
\hline $\mathrm{SC} 1$ & 1.0 & 0.009648 & 270.0 & 348.16 & 0.9529 & 180.0 \\
\hline $\mathrm{SC} 2$ & 1.0 & 0.009648 & 270.0 & 108.16 & 0.9529 & 60.0 \\
\hline SC3 & 1.0 & 0.009648 & 270.0 & 228.16 & 0.9529 & 300.0 \\
\hline
\end{tabular}
$\theta_{0}=-23^{\circ}$, and other elements are the same as in Example 1 for the starting orbits.

Table 3. Example 1: starting orbits, epoch 2457023.5

Table 4. Example 1: Optimized orbits, epoch 2457023.5.

\begin{tabular}{cccccrr}
\hline & $\mathrm{a}$ & $\mathrm{e}$ & $\omega$ & $\Omega$ & $\mathrm{I}$ & \multicolumn{1}{c}{$\mathrm{M}$} \\
\hline $\mathrm{SC} 1$ & 0.9999840 & 0.0095697524 & 269.308799 & 348.052593 & 0.95264106 & 180.780276 \\
$\mathrm{SC} 2$ & 0.9999941 & 0.0095545041 & 270.296900 & 107.898193 & 0.95272462 & 59.958911 \\
SC3 & 1.0000004 & 0.0096671214 & 269.592620 & 228.372943 & 0.95293639 & 300.188236 \\
\hline
\end{tabular}


Table 5. Example 1: Initial positions and velocities at epoch 2457023.5.

(J2000.0 solar-system-barycentric equatorial coordinates, in AU and AU/day.)

\begin{tabular}{ccccccc}
\hline & $x$ & $y$ & $z$ & $\dot{y}$ & $\dot{z}$ \\
\hline SC1 & 0.21052214 & 0.89889830 & 0.40786493 & -0.016670363 & 0.0032193213 & 0.0013952001 \\
SC2 & 0.19085381 & 0.89912108 & 0.38098013 & -0.016938360 & 0.0030315238 & 0.0015874632 \\
SC3 & 0.22353943 & 0.89276563 & 0.37821601 & -0.016880581 & 0.0034893057 & 0.0012394541 \\
\hline
\end{tabular}

Table 6. Example 1: variation range of the 3 arm-lengths and trailing argument before optimization.

\begin{tabular}{lcccc}
\hline & Maximum & Minimum & Average & Range of Variation \\
\hline $\mathrm{SC} 1 / \mathrm{SC} 2(\mathrm{~km})$ & 5105704 & 4893333 & 4999518.5 & 212371 \\
$\mathrm{SC} 2 / \mathrm{SC} 3(\mathrm{~km})$ & 5057007 & 4940377 & 4998692 & 116630 \\
$\mathrm{SC} 3 / \mathrm{SC} 1(\mathrm{~km})$ & 5218296 & 4782349 & 5000322.5 & 435947 \\
$\theta$ (degree) & 29.5 & 20.1 & 24.8 & 9.4 \\
\hline
\end{tabular}

Table 7. Example 1: variation range of the 3 arm-lengths and trailing argument after optimization.

\begin{tabular}{lcccc}
\hline & Maximum & Minimum & Average & Range of Variation \\
\hline SC1/SC2 $(\mathrm{km})$ & 5027287 & 4934658 & 4980972.5 & 92629 \\
$\mathrm{SC} 2 / \mathrm{SC} 3(\mathrm{~km})$ & 5027076 & 4935075 & 4981075.5 & 92001 \\
$\mathrm{SC} 3 / \mathrm{SC} 1(\mathrm{~km})$ & 5021496 & 4928770 & 4975133 & 92726 \\
$\theta$ (degree) & 29.4 & 20.1 & 24.8 & 9.3 \\
\hline
\end{tabular}

The optimized orbit elements are shown in Table 8. The initial positions and velocities of the three spacecraft are shown in Table 9 . The variation range of the three arm-lengths and the trailing angle before and after optimization are shown in Tables 10 and 11.

Example 3. In order to further reduce the variation range of the trailing angle, the semi-major axis $a$ is decreased to $0.9992, \theta_{0}=-23^{\circ}$, and other elements are

Table 8. Example 2: optimized orbits, epoch 2457023.5.

\begin{tabular}{rccccrr}
\hline & $\mathrm{a}$ & $\mathrm{e}$ & $\omega$ & $\Omega$ & $\mathrm{I}$ & \multicolumn{1}{c}{$\mathrm{M}$} \\
\hline SC1 & 0.99958503 & 0.0096474586 & 269.216275 & 347.545313 & 0.95288002 & 180.381125 \\
SC2 & 0.99959814 & 0.0095545707 & 270.620534 & 107.197396 & 0.95268515 & 60.346346 \\
SC3 & 0.99960184 & 0.0096285612 & 269.203019 & 227.192554 & 0.95297519 & 299.761583 \\
\hline
\end{tabular}

Table 9. Example 2: Initial positions and velocities at epoch 2457023.5.

(J2000.0 solar-system-barycentric equatorial coordinates, in AU and AU/day.)

\begin{tabular}{ccccccc}
\hline & $x$ & $y$ & $z$ & $\dot{x}$ & $\dot{y}$ & $\dot{z}$ \\
\hline SC1 & 0.22751260 & 0.89517572 & 0.40624925 & -0.016609014 & 0.0034839976 & 0.0015125815 \\
SC2 & 0.20757538 & 0.89574361 & 0.37944055 & -0.016879487 & 0.0032998238 & 0.0017030419 \\
SC3 & 0.24031306 & 0.88884462 & 0.37646786 & -0.016815840 & 0.0037585330 & 0.0013566281 \\
\hline
\end{tabular}


Table 10. Example 2: variation range of 3 arm-lengths and trailing argument before optimization.

\begin{tabular}{ccccc}
\hline & Maximum & Minimum & Average & Range of Variation \\
\hline $\mathrm{SC} 1 / \mathrm{SC} 2(\mathrm{~km})$ & 5099951 & 4895767 & 4997859 & 204184 \\
$\mathrm{SC} 2 / \mathrm{SC} 3(\mathrm{~km})$ & 5056910 & 4937033 & 4996971.5 & 119877 \\
$\mathrm{SC} 3 / \mathrm{SC} 1(\mathrm{~km})$ & 5214379 & 4783056 & 4998717.5 & 431323 \\
$\theta$ (degree) & 28.1 & 20.9 & 24.5 & 7.3 \\
\hline
\end{tabular}

Table 11. Example 2: variation range of the 3 arm-lengths and trailing argument after optimization.

\begin{tabular}{ccccc}
\hline & Maximum & Minimum & Average & Range of Variation \\
\hline $\mathrm{SC} 1 / \mathrm{SC} 2(\mathrm{~km})$ & 5029112 & 4931847 & 4980479.5 & 97265 \\
$\mathrm{SC} 2 / \mathrm{SC} 3(\mathrm{~km})$ & 5035682 & 4937844 & 4986763 & 97838 \\
$\mathrm{SC} 3 / \mathrm{SC} 1(\mathrm{~km})$ & 5033871 & 4937680 & 4985775.5 & 96191 \\
$\theta$ (degree) & 28.1 & 20.9 & 24.5 & 7.3 \\
\hline
\end{tabular}

Table 12. Example 3: optimized orbits, epoch 2457023.5.

\begin{tabular}{ccccccc}
\hline & $\mathrm{a}$ & $\mathrm{e}$ & $\omega$ & $\Omega$ & $\mathrm{I}$ & $\mathrm{M}$ \\
\hline $\mathrm{SC} 1$ & 0.99918332 & 0.0095398673 & 269.457352 & 346.528674 & 0.953282441 & 180.153419 \\
$\mathrm{SC} 2$ & 0.99919252 & 0.0095930589 & 269.686204 & 106.171473 & 0.953116093 & 60.292415 \\
$\mathrm{SC} 3$ & 0.99920044 & 0.0096328472 & 269.713106 & 226.149645 & 0.952912432 & 300.291941 \\
\hline
\end{tabular}

still unchanged. The optimized orbit elements are shown in Table 12. The initial positions and velocities of the three spacecraft are shown in Table 13. The variation range of the three arm-lengths and the trailing angle before and after optimization are shown in Table 14 and Table 15.

In this example the variations of the parameters are shown as Fig. 11.

Table 13. Example 3: Initial positions and velocities at epoch 2457023.5.

(J2000.0 solar-system-barycentric equatorial coordinates, in AU and AU/day.)

\begin{tabular}{ccccccc}
\hline & $x$ & $y$ & $z$ & $\dot{x}$ & $\dot{y}$ & $\dot{z}$ \\
\hline SC1 & 0.24450548 & 0.89098869 & 0.40443241 & -0.016545313 & 0.0037509529 & 0.0016282385 \\
SC2 & 0.22462322 & 0.89190479 & 0.37778021 & -0.016815330 & 0.0035737736 & 0.0018220357 \\
SC3 & 0.25697908 & 0.88449335 & 0.37457374 & -0.016748463 & 0.0040284244 & 0.0014736968 \\
\hline
\end{tabular}

Table 14. Example 3: variation range of 3 arm-lengths and trailing argument before optimization.

\begin{tabular}{ccccc}
\hline & Maximum & Minimum & Average & Range of Variation \\
\hline $\mathrm{SC} 1 / \mathrm{SC} 2(\mathrm{~km})$ & 5120898 & 4873729 & 4997313.5 & 247169 \\
$\mathrm{SC} 2 / \mathrm{SC} 3(\mathrm{~km})$ & 5064827 & 4926894 & 4995860.5 & 137933 \\
$\mathrm{SC} 3 / \mathrm{SC} 1(\mathrm{~km})$ & 5240406 & 4752139 & 4996272.5 & 488267 \\
$\theta$ (degree) & 26.3 & 20.3 & 23.3 & 6.0 \\
\hline
\end{tabular}


Table 15. Example 3: variation range of 3 arm-lengths and trailing argument after optimization.

\begin{tabular}{lcccc}
\hline & Maximum & Minimum & Average & Range of Variation \\
\hline SC1/SC2 $(\mathrm{km})$ & 5002139 & 4909196 & 4955667.5 & 92943 \\
SC2/SC3 $(\mathrm{km})$ & 5030928 & 4938200 & 4984564 & 92728 \\
SC3/SC1(km) & 5025157 & 4931006 & 4978081.5 & 94151 \\
$\theta$ (degree) & 26.8 & 21.2 & 24.0 & 5.6 \\
\hline
\end{tabular}
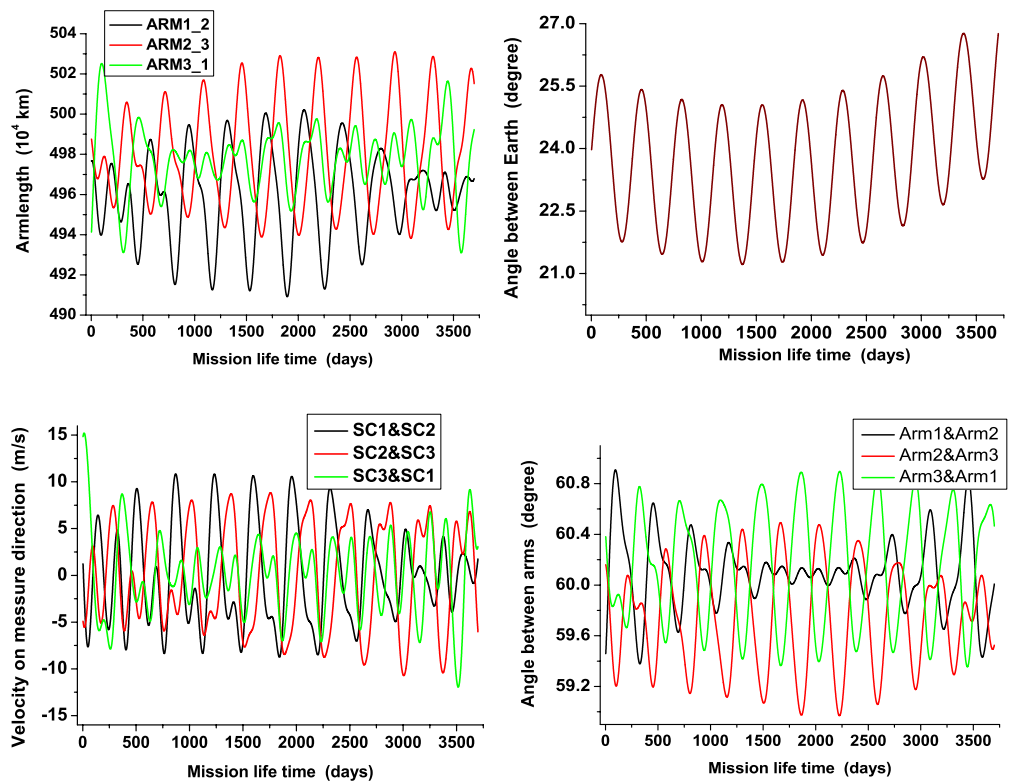

Fig. 11. Example 3: the variations of the arm-lengths, trailing angle, velocities in the measurement direction and the angles between arms.

\section{Acknowledgments}

We would like to thank the Foundation of Minor Planets of Purple Mountain Observatory and the National Science Foundation (Grant No. 10503013) for their financial support. We would also like to thank Professor Karsten Danzmann at the Max Planck Institute for Gravitational Physics and Professor Wei-Tou Ni at the Purple Mountain Observatory especially for their great support to this work.

\section{References}

1. LISA Pre-Phase A Report, second edition, Max-Planck-Institut für Quantenoptik, Report 233 (July 1998); often referred to as PPA2.

2. A. Milani, A. M. Nobili and P. Farinella, Non Gravitational Perturbations and Satellite Geodes (Adam Hilger Ltd., Bristol and Boston, 1987).

3. A. J. Sanders and G. T. Gillies, A Comparative Study of Proposals for Space-Based Determination of the Gravitational Constant G, Rivista del Nuovo Cimento 19(2) (1996) 1-54. 
4. D. Brouwer and G. M. Clemence, Methods of Celestial Mechanics, (Academic Press, New York, London, 1961).

5. J. E. Faller, P. L. Bender, J. L. Hall, D. Hils and M. A. Vincent, Space antenna for gravitational wave astronomy Kilometric, Optical Arrays in Space, Proc. Colloquium (Cargese, Corsica, France 23-25 Oct. 1984) European Space Agency, SP-226 (1985).

6. M. A. Vincent and P. L. Bender, Proc. Astrodynamics Specialist Conf. (Kalispell), Vol. 1 (Univelt San Diego. CA), (1987) 1346.

7. W. M. Folkner, F. Hechler, T. H. Sweetser, M. A. Vincent and P. L. Bender, LISA orbit selection and stability, Class. Quantum Grav. 14 (1997) 1405.

8. C. Cutler, Phys. Rev. D 57 (1998) 7089.

9. S P. Hughes, Preliminary optimal orbit design for laser interferometer space antenna, 25th Annual AAS Guidance and Control Conference, Breckenridge CO, Feb. (2002).

10. F. Hechler and W.M. Folkner, Adv. Space Res. 32 (2003) 1277.

11. S. V. Dhurandhar, K. Rajesh Nayak, S. Koshti and J.-Y. Vinet, Class. Quant. Grav. 22 (2005) 481.

12. T. H. Sweetser, Class. Quant. Grav. 22 (2005) S429.

13. K. Rajesh Nayak, S. Koshti, S. V. Dhurandhar and J.-Y. Vinet, Class. Quant. Grav. 23 (2006) 1763.

14. W.-T. Ni et al., Acta Astronautica 59 (2006) 598.

15. W.-T. Ni, Int. J. Mod. Phys. D 17 (2008) 921 [arXiv: 0712.2492] and references therein.

16. C.-J. Tang, C.-H. Chang, W.-T. Ni and A.-M. Wu, Orbit Design for ASTROD I, report (2003).

17. Y. Xia, G. Li and W.-T. Ni, Orbit design and orbit simulation for ASTROD I for the 2012 and 2013 launch windows, Electronic Proceedings of the 15th Workshop on JAXA Astrodynamics and Flight Mechanics, Kanagawa, Japan, July 25-26, (2005).

18. Z. Yi, G. Li, G. Heinzel, A. Rüdiger, O. Jennrich, L. Wang, Y. Xia, F. Zeng and H. Zhao, Coorbital Restricted Problem and its Application in the Design of the Orbits of the LISA Spacecraft, Int. J. Mod. Phys. D 17 (2008) 1005.

19. C. D. Murray and S. F. Dermott, Solar System Dynamics, (Cambridge University Press, 1999).

20. L. Anselmo, B. Bertotti, P. Farinella, A. Milani and A. M. Nobili, Orbital perturbations due to radiation pressure for a spacecraft of complex shape, Celestial Mechanics 29 (1983) 27-43.

21. Alvin J. Sanders and W. E. Deeds, Proposed new determination of the gravitational constant G and tests of Newtonian gravitation, Phys. Rev. D 46 (1992) 489-504.

22. R. R. Newton, Stabilizing a spherical satellite by radiation pressure, Am. Rocket Soc. J. 30(12) (1960) 1175-1177.

23. A. J. Sanders, W. E. Deeds and M. E. Rupright, Method for producing extreme microgravity in extended volumes, US Patent 5, 887, 827, March 30, 1999.

24. P. Farinella, A.M. Nobili, F. Barlier and F. Magnard, Effects of thermal thrust on the node and inclination of LAGEOS, Astron. Astrophys. 234(1-2) (1990) 546-554.

25. R. Battiti and G. Techiolli, The Continuous Reactive Tabu Search: Blending Combinatorial Optimization and Stochastic Search for Global Optimization (Istituto Trentino Di Cultura, 1994).

26. G. Li, W.-T. Ni and L. Tian, PMOE 2003 Planetary Ephemeris Framework (I) Mathematical Model, Publications of Purple Mountain Observatory, 22, (3-4) (2003) 12, in Chinese.

27. G. Li and L. Tian, PMOE 2003 Planetary Ephemeris Framework (II) Integrators and Programs, Publications of Purple Mountain Observatory, 22 (3-4) (2003) 32, in Chinese.

28. P. Herget, The Computation of Orbits, (Cincinnati Observatory, 1948). 